\title{
Ontological Visualization of Knowledge Structures Based on the Operational
}

\author{
Management of Information Objects
}

\begin{abstract}
The properties of ontological models to reflect the conceptual view of a researcher on a certain subject area and uniquely determine its concept, structure, accumulate and reuse knowledge repeatedly and make it possible to widely use it in the educational process. However, the existing tools for constructing ontological models of ontology knowledge (Protégé in integration with OWLViz, OntoGraf, 3D Hyperbolyc, Tree, TODOS, etc.) only partially satisfy the needs of the educational process. Currently, many methods and tools for visualizing ontologies are used. The choice of a particular visualization method is specific and has its own characteristics depending on the task. When choosing visualization tools, it is important that they do not only help to effectively reflect all the information, but, at the same time, allow the user to perform easily various operations on ontologies. There is no single definite method that is universal. So, a viable solution is to provide the user with several visualization options so that it is able to choose the one that is suitable for its current needs. The article describes the author's web resource http://ontos.xyz, designed to create and visualize the hierarchical structure of subject areas using an ontograph. An ontograph is implemented as a combination of content-containing concepts (vertices, nodes) and the relationships between these concepts. The authors determine the possibilities of using the ontological approach in the pedagogical process by the example of working with the web resource they created, which provides convenient use of ontologies. The article discusses examples of using ontologies to provide semantic annotation for collections of images, sound and other non-text objects; for the development of documentation of arbitrary volume; for building directories with the ability to support the surjective connection of objects (nodes); to simplify the performance of search work, etc. The purpose of the article is to reveal the features of the use of ontological structures for visualizing knowledge schemes and components of the operational management of information objects.
\end{abstract}

Keywords: ontologies, knowledge bases, remote education

\section{$1 \quad$ Problem statement}

Knowledge is an integral part of any educational process. In connection with the current situation in the world related to the viral pandemic, the question of introducing remote forms of education is extremely acute. Their usage involves the indirect interaction of a teacher and a student with the help of many forms of communication provided by the capabilities of modern Internet technologies.

In connection with the current situation in the world related to the viral pandemic, the question of introducing remote forms of education is extremely acute. The use of 
remote forms of education involves the indirect interaction of a teacher and a student with the help of many forms of communication provided by the capabilities of modern Internet technologies. Consequently in recent years, there has been a growing interest in semantic technologies that provide the ability to implement automated processing of conceptualized knowledge [1,2]. An important component of such technologies is the technology of ontological representation of knowledge, which allows the creation of formalized electronic models of knowledge through ontological modeling.

\section{$2 \quad$ Analysis of recent research and publications}

Today, there are many definitions of the concept of "ontology" to varying degrees, reflecting the semantic content of this phenomenon. In the framework of this work, we will use the definition proposed in 1996 by van Heinst and his colleagues: "An ontology is an explicit knowledge-level specification of a conceptualization, e.g. the set of distinctions that are meaningful to an agent. The conceptualization - and therefore the ontology - may be affected by the particular domain and the particular task it is intended for" [3].

\section{Statement of basic material and the substantiation of the obtained results}

The properties of ontological models to reflect the conceptual view of a researcher on a certain subject area and uniquely determine its concepts, structure, accumulate and repeatedly use knowledge allow them to be widely used in the educational process. Obviously, any subject area is a subset of the general information space - the universe.

It is indicated by the symbol $\mathrm{U}$ in the constructed diagram. The subject area $\mathrm{P}$, which is a subset of the universe, defines a certain scientific theory, the study of a certain part of which should be provided by the textbook T. The part (subset) of the subject area, the study of which is intended to provide the textbook does not limit the content of the textbook to 100 percent. To describe the concepts of subject domain $\mathrm{P}$, the textbook should use data from other subject areas (let's call them auxiliary), which, in turn, are subsets of the universum (Fig. 1).

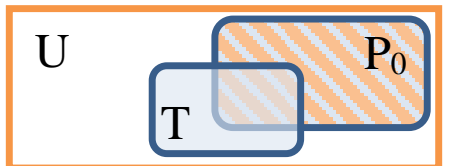

Fig. 1 - Venn Diagram of the Sets of the Textbook $\mathrm{T}$ and the Subject Area $\mathrm{P}_{0}$ Studied With its Help

These areas include the concepts of related and serving sciences. For example, consider the second Faraday law. He determines that for a certain amount of electricity, the mass of the chemical element formed on the electrode is directly 
proportional to the equivalent mass of the element. It is equal to the mass of one equivalent - the amount of substance that reacts or replaces 1 mole of hydrogen atoms in chemical reactions: $\mu_{\mathrm{eq}}=\mu / \mathrm{z}$, where:

$\mu$ is the molar mass of the substance;

$\mathrm{z}$ is the number of electrons per ion (valence number of ions).

The concept of molar mass is studied at chemistry lessons and refers to the subject areas of both educational subjects (both chemistry and physics). The mathematical actions that are used to describe the formula representation of laws are determined by mathematical terms, the order of presentation of the material, its content is determined by laws (and, therefore, the terms of didactics), etc.

Another example of the usage of different subject ontologies within the same textbook is a retrospective of the history of various discoveries, fragments of biographies of scientists, etc.

Some authors use the concept of metaontology, meaning by this concept either a kind of "connecting" ontology, for example, an ontology of the "human" language for describing a subset area, or an ontology for which domain ontologies used to solve a particular problem are subsets. So, for example, G. Evgenev indicates that metaontology operates with general concepts and relationships that are independent of a specific subject area. The subject ontology contains concepts that describe a specific subject area and the relationship between them. The task ontology contains functions with the help of which the input data are converted into output data [4].

Thus, the textbook can be represented as the intersection of the sets of terms of interconnected subject areas $\left(\mathrm{P}_{\mathrm{i}}, \mathrm{i}=\overline{1, n}\right)$.

$$
\mathrm{T}=\bigcap_{1}^{n} P_{i} \neq \varnothing
$$

Let us represent each of the subject areas used in constructing the textbook in the form of an ontological model. To do this, we describe the ontology by mathematical methods. A formal description of an ontology in the form of a triple is considered classical [5].

$\mathrm{O}=\langle\mathrm{X}, \mathrm{R}, \mathrm{F}\rangle$, where $\mathrm{O}$, in fact, ontology;

$\mathrm{X}=\left\{\mathrm{x}_{1}, \mathrm{x}_{2}, \ldots \mathrm{x}_{\mathrm{n}}\right\}$ is a finite set of domain concepts, and $\mathrm{n}$ is the number of elements in this set;

$R=\left\{r_{1}, r_{2}, \ldots r_{m}\right\}$ - a finite set of relations between the concepts of the subject area, $\mathrm{m}$ - the number of significant relations;

F- is a finite set of interpretation functions defined on concepts and ontology relations $\mathrm{O}[6,7] . \mathrm{F}$ is the Cartesian product $\mathrm{X} \times \mathrm{R}$ is a finite set of interpretation functions defined on concepts and / or relationships.

In the general case, relations can be conditionally divided into generally significant (of which, as a rule, relations of a partial order are distinguished) and specific relations of a given subject area. In fact, the relation $\mathrm{R}$ is an interpretation of the properties of concepts, that is, there is a transformation that each relation establishes a correspondence of a certain property.

Let us depict the triplet graphically. In the boundary case, $n=1$, respectively, the number of significant relations in the set $R$ (taking into account the void $X \times R$ ) equals to 1 , and the triplet corresponding to such an ontology can be uniquely determined using a simple ontograph (Fig. 2). 


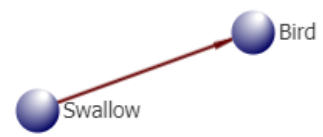

Fig. 2 - The simplest Ontograph

Since the relation uniquely determines the dependencies of concepts, the edge of such an ontograph always represents a directed segment.

In the ontograph depicted, the "Swallow" peak is a daughter, with respect to the "Bird" peak. In this ontograph, the relationship defines the standard relation "is", or, in other words, "one of the elements of the set". Building an ontological model allows to specify other dependencies, for example, expressed by the words "more than" or "coincides with an accuracy of similarity coefficient."

Thus, a combination of the simplest ontographs, between which nodes dependencies are also defined, allows you to build an ontological model of any complexity.

Currently, there are numbers of tools for creating and supporting ontologies that, in addition to the general editing and viewing functions, support ontology documentation, import and export of ontologies of various formats and languages, support graphic editing, manage ontology libraries, etc. [8].

Obviously, the manual should provide an explicit statement of knowledge in its subject area, and therefore the use of scientifically based methodology, such as ontology, is necessary [9].

One of the most widespread, and therefore demanded, is the development of Stanford Center for Biomedical Informatics Research at Stanford University School of Medicine called Protégé. Protégé is a tool that allows to create a domain's ontology, custom data entry forms for data entry. Protégé allows to define classes, class hierarchies, variables, variable constraints, and relationships between classes and the properties of these relationships.

Platform-integrated applications allow ontologies to be displayed in various ways (Fig. 3).

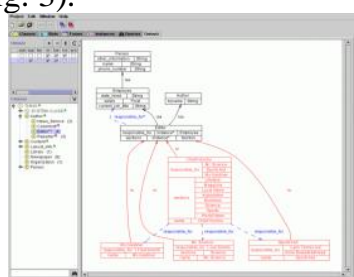

a)

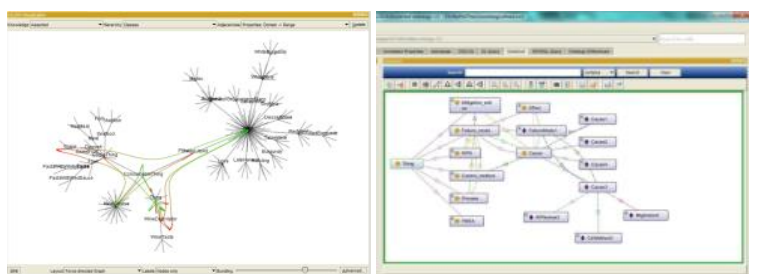

b) c)

Fig. 3 - Ontology Tools for Displaying in Protégé: a) OntoViz; b) GlowViz; c) OntoGraph

The existing ontology construction tools are not limited by the Protégé platform and only partially satisfy the needs of the educational process.

So in the line of visualization tools you can select 3D Hyperbolyc Tree -it builds an ontograph tree in hyperbolic space; Conceptino - is a visual graph designer that 
organizes large amounts of information into graphic schemes; tools for creating "flat trees", for example, the resource https://www.anychart.com; SciVi scientific visualization system with the installed CGraph application (Fig. 4)

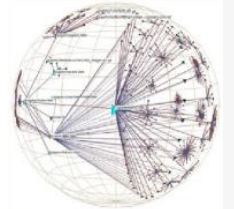

a)

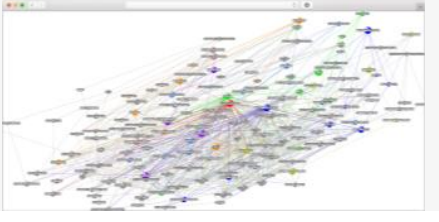

b)

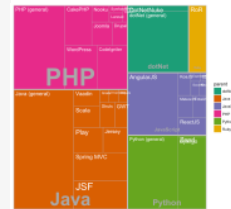

c)

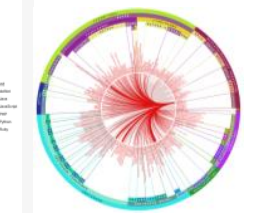

d)

Fig. 4 - Visualization Tools for Ontographs:a) 3D Hyperbolyc Tree; b) Conceptino; c) Anychart; d) SciVi-Cgraph

And many other means of visual display of ontologies, the review of which is beyond the scope of the material considered in our article, due to limited volume.

All these visualizations have one common specific feature, which is often very convenient, and even necessary for research: the relationships between all objects are displayed (or all objects are shown at the same time, as in Anychart), but, as we can see, all the proposed implementations ontology mappings are quite far from the kind of electronic textbook familiar to participants in the educational process.

Unfortunately, in relation to the educational process, this approach is far from being always justified. With a sufficiently large number of connections (typical, for example, for school textbooks), the screen becomes cluttered with elements that are unnecessary for immediate work and, therefore, elements that are harmful to the perception of educational material.

Let us give an example of an image of sufficiently large ontographs with obvious redundancy in the display of elements (Fig. 5).
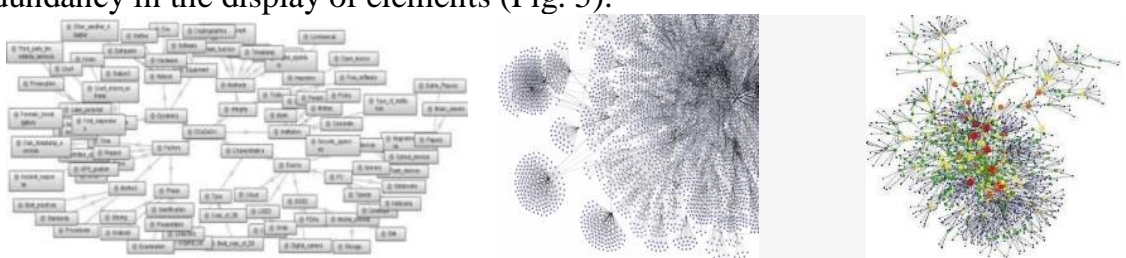

Fig. 5 - Examples of Displaying Ontographs with a Large Number of Connections

One of the tools of visualizing ontological models, where a breakthrough in improving usability was made, was the development by VAH of Ukraine of TODOS (TODOOS) - a system with a powerful user interface focused on various research and other tasks [10].
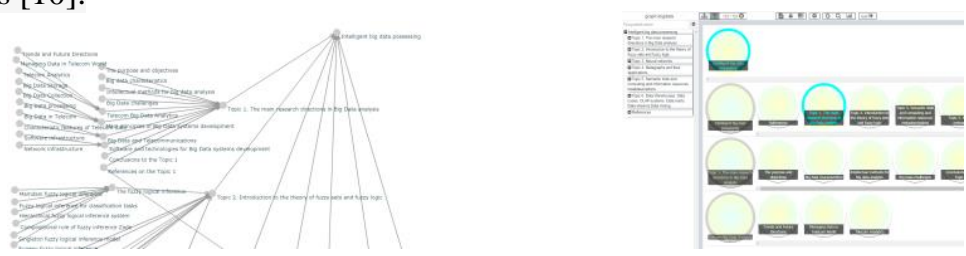

Fig. 6 - Various Variants of the TODOS User Interface 
One of the successful solutions of the problem of clutter of visualization was the development of the Minor Academy of Sciences of Ukraine - the ontological prism presented in the systems TODOS (Fig. 7) and AgrOn [11].

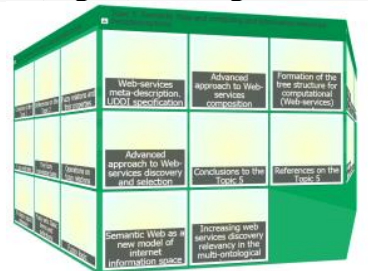

Fig. 7 - Ontoprism in Todos System

Being one of the most convenient tools for displaying an electronic textbook, ontoprism is a special case of the visualization system, which consists of two components: a navigator that determines the path to some node of the ontograph that has child elements and a visualization slider for this node that displays these elements. When one clicks on the display of an element on the edge of the prism, a pop-up window for the content of each element appears, which can contain text, a picture or a hyperlink.

A navigator can be an ontograph, a tree, navigation buttons, hyperlinks, etc. Its main goal is to determine the node which visualization will be carried out.

Indeed, when working with a familiar textbook of the "ordinary" or "paper" type, a participant in the pedagogical process, as a rule, does not constantly observe the path to the current page (content) of the textbook.

While agreeing with the proposed approach, though we feel like to note that the proposed platform, being a research tool, does not fully meet the needs of the educational process. First of all, it is not provided on the pages of arbitrary formatting content, there is no possibility of introducing feedback, there is no compatibility with web 2.0 technologies, etc., without which it is difficult to imagine a modern electronic textbook.

Based on the foregoing, the task arose: to implement an ontological approach in relation to the actual creation of an electronic textbook that would correspond to modern capabilities.

To achieve this goal, an online resource http://ontos.xyz/ (Fig. 8) was developed consisting of an editor and a viewer.

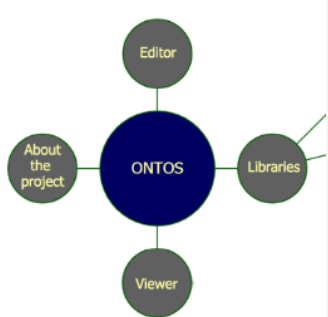

a)

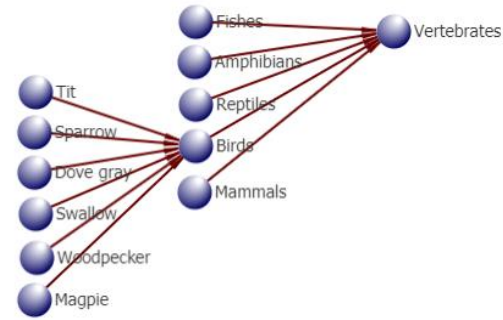

b)

Fig. 8 - Ontos Project: a) the main page; b) an example of an ontograph 
Like many systems, the editor is designed to create an ontograph. The main feature of the editor is the ability to assign each node a context of all types supported by the browser. Including html-pages, web 2.0 resources, etc. (Fig. 9 a). The second distinguishing feature of the development is the maximum approximation of the displayed node to the familiar textbook (Fig. 9b).

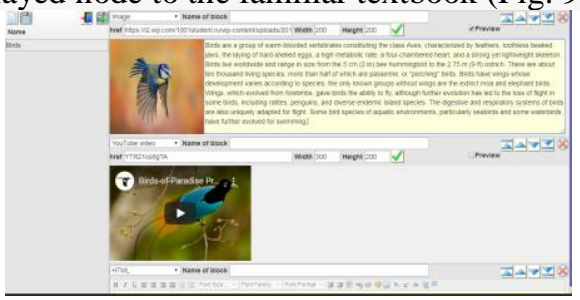

a)

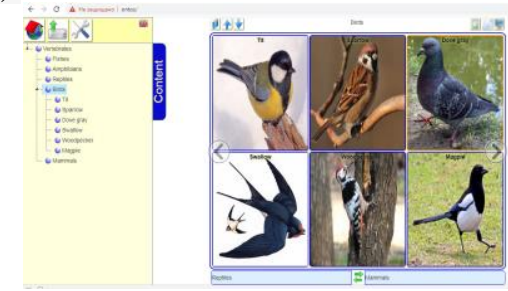

b)

Fig.9 - Context Creation Management Window

The fully implemented principle of the two-component ontograph display technology is the presence of content with hyperlinks to transitions to individual vertices and, separately, a navigation system with transitions to neighboring (single-level, parent and child) nodes.

\section{Conclusions}

With the advent of the semantic network and semantic technologies, ontologies have emerged that have become one of the most sought after paradigms for representing knowledge. Ontological models reflect the conceptual view of a researcher on a certain subject area and provide an opportunity to uniquely determine its concept, structure, accumulate and repeatedly use knowledge. A number of scientists define the following ontology functions in education: simplification due to automatization and systematization of students' search for information on the chosen research topic; providing students with the opportunity to analyze and compare information within the chosen topic due to the clear structuring of information; the development of new information by students on the basis of knowledge gained for further use in independent research; activation and effective use by students information resources of a society about a given subject area; expanding one's worldview, as it simplifies the opportunity to get acquainted with related concepts that go beyond the standard course; increasing student motivation to study. Presentation of information in the form of an ontological model with a two-component display allows to display individual information concepts (terms, concepts) in a convenient form for the participant in the educational process and get a lot of their semantic connections with other objects, thereby understanding their role in this knowledge system or in solving the problem.

The ontological approach to education cannot be just a means of organizing knowledge. Expanding the traditional functions of software that works with ontologies, one can create an information environment in which active work with knowledge is provided, and educational tasks are also solved in an original way. 


\section{References}

1. Zupanc, K., \& Bosnić, Z. (2017). Automated essay evaluation with semantic analysis. $\quad$ Knowledge-Based $\quad$ Systems, $\quad 120, \quad 118-$ 132.https://doi.org/10.1016/j.knosys.2017.01.006

2. Kovalenko O., Bondarenko T., Kovalenko D. (2019) Evaluation Automation of Achievement Tests Validity Based on Semantic Analysis of Training Texts. In: Auer M., Tsiatsos T. (eds) The Challenges of the Digital Transformation in Education. ICL 2018. Advances in Intelligent Systems and Computing, vol 917. Springer, Cham. PP. 485-492.

3. Van Heijst, G., Schreiber, A. T., and Wielinga, B. J. (1996). Using Explicit Ontologies in KBS Development. International Journal of Human and Computer Studies(in press).

4. Evgenev G. B. (2014) Ontological Methodology for Creating Textbooks / Engineering Bulletin, September, No. 09.

5. Norenkov I.P. (2010). Intelligent Technologies Based on Ontologies. // Information Technology. No. 1. S. 17-24.

6. Palagin A.V., Yakovlev Yu.S. (2005). System Integration of Computer Technology. - Vinnitsa: UNIVERSUM. $680 \mathrm{~s}$

7. Katifori, C. (2007). Halatsis. Ontology Visualization Methods - a Survey, ACM $\begin{array}{llllll}\text { Computing } & \text { Surveys } & \text { (CSUR). } & \text { Volume } & 39 & \text { Issue }\end{array}$ Access:http://www.dit.unitn.it/ p2p/RelatedWork/Matching/a10-katifori.pdf.

8. Greger Sergey Eduardovich (2012). Editor of the Ontological System Metamodel // Object Systems. No. 1 (6). URL: https://cyberleninka.ru/article/n/redaktormetamodeli-ontologicheskoy-sistemy.

9. Nesterenko O., Trofymchuk O. (2019). Patterns in Porming the Ontologybased Environment of Informationanalytical Activity in Administrative Management // Information Technologies. Management Systems in Industry. -Vol. 5, No. 2 (101). Access: http://journals.uran.ua/eejet/article/view/180107/182267.

10. Stryzhak O.E. (2017). TODOS - IT-Platform for the Formation of Transdisciplinary Information Environments / V.Yu. Velychco, M.A. Popova, V.V. Prikhodnyuk, O.E. Stryzhak. - Weapons Systems and Military Equipment. - № 1 (49). - p. 10-19.

11. Guraliuk A.G. (2018). Modern Means of Visualization of Subject Ontologies on Adaptive Principles [Electronic resource] / A.G. Guraliuk, M.L. Rostoka // Adaptive Management: Theory and Practice. Series "Pedagogy". — № 4 (7). Access: https://amtp.org.ua/index.php /journal/article/download/10/6/. 